\title{
Development of Metal Containing Polymers For Optoelectronic Applications
}

\author{
Wai Kin Chan*, Sijian Hou, Po King Ng, Chi Tak Wong, Sze Chit Yu \\ Department of Chemistry, University of Hong Kong, Pokfulam Road, Hong Kong, China
}

\begin{abstract}
Most of the work in organic electroluminescent polymers has been focused on organic conjugated polymers. However, polymers attached with transition metal complex have received relatively less attention. We have synthesized and studied the light emitting properties of some metal containing polymers based on the polypyridine complexes of rhenium and ruthenium. These complexes exhibit long-lived excited states caused by the metal to ligand charge transfer (MLCT) transitions. By varying the structure of the ligand and/or the transition metal, we are able to fine-tune the electronic properties of the resulting metal complexes.

We have synthesized a series of poly(phenylenevinylene) (PPV) derivatives which are functionalized with ruthenium polypyridine complexes at the polymer mainchain or side chain. These complexes are able to act as photosensitizers which enhance the photoconductivity of these polymers at longer wavelength. Both the conjugated backbone and the metal complex can emit light upon excitation. As a result, it is possible to tune the color by loading different amount of ruthenium complex to the polymer. Luminescence studies showed that the ruthenium complex could quench the emission of the conjugated backbone in some polymers, which suggests an energy transfer process between the backbone and the metal complexes. It was also found that the presence of metal complexes could enhance the charge carrier mobilities of the polymers, as the metal and/or ligands can act as extra charge carriers in the charge transport process.
\end{abstract}

Keywords: poly(p-phenylenevinylene), PPV, ruthenium, bipyridine, terpyridine, light emitting device

\section{INTRODUCTION}

Since the first report of electroluminescence (EL) in poly(p-phenylenevinylene) (PPV) ${ }^{1}$ different examples of EL devices based on a variety of materials including conjugated polymers ${ }^{2-3}$ and fluorescent dye-doped polymeric systems $\mathrm{s}^{4-5}$ have been reported. The use of transition or rare earth metal complexes for EL devices ${ }^{6-8}$ has been gaining more attention because of the flexibility in structure design and modification. In normal organic materials, the maximum quantum efficiency for an EL process originated from the emission of singlet excitons is $25 \%$ due to the spin statistic for the production of singlet and triplet excited states by charge recombination. This limits the quantum efficiency and performance of the EL devices based on ordinary organic molecular or polymeric materials.

Our group has developed different types of conjugated polymers functionalized with ruthenium (II) bipyridyl (bpy) or terpyridyl (tpy) complexes. ${ }^{9-13}$ The chemistry and photophysics of ruthenium bipyridyl ${ }^{14-15}$ and terpyridyl $1^{16-18}$ complexes have been studied extensively. They exhibit a relatively long-lived metal-to-ligand charge transfer (MLCT) excited state which makes it a promising candidate for EL devices. Upon excitation, the singlet excited state undergo intersystem crossing to the lowest triplet ${ }^{3}$ MLCT excited states and the quantum yield for this process is essentially unity. This triplet excited state is able to emit light at around $600-650 \mathrm{~nm}$ depending on the nature of the bipyridine ligand. By utilizing the emission from the triplet states, it is possible to exceed the theoretical $25 \%$ limit for traditional organic light emitting devices. ${ }^{19}$ In addition, the ruthenium polypyridine complexes exhibit a reversible ruthenium

• Correspondence Email: waichan@hkucc.hku.hk; Telephone: (852) 2859-8943; Fax: (852) 2857-1586 
centered $\mathrm{Ru}^{\mathrm{II} / \mathrm{III}}$ oxidation and several other ligand-based reductions. As a result, the ruthenium complexes may also play the role as charge transporting species and thus enhance the charge carrier mobilities, which is very important to the performance of EL devices.

Fine tuning of the light emitting properties can be achieved by modifying the structure of the ligand or by using different transition metals. There are several approaches to incorporate transition metal complexes to the polymer molecule. In the first approach, the metal center constitutes part of the polymer mainchain (Figure 1). The second approach involves the use of the polymer mainchain as the site for coordination. Alternatively, the metal complexes can also be attached to the polymer molecule as pendant group. In these polymers, there are two light emitting moieties: the $\pi$-conjugated main chain and the ruthenium complexes. Usually the PPV mainchain emits in the green region while the ruthenium complex emits in the red region. Therefore, the emission color is a combination of these two colors. Tuning of the emission properties can be adjusted by changing the metal content in the polymers.

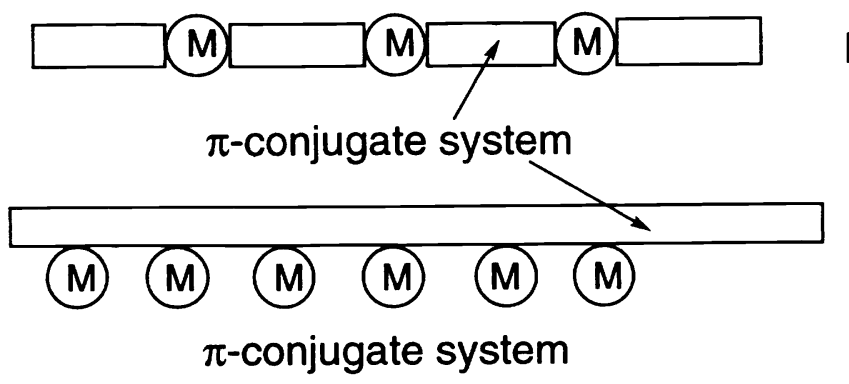

Metal center on the mainchain

Metal coordinated attached to the mainchain

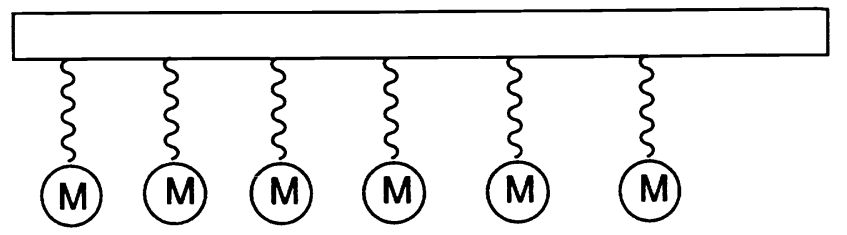

Metal complex attached as pendant chain

Figure 1. Design of different types of metal containing polymers.

\section{RESULTS AND DISCUSSION}

The ruthenium containing PPVs were synthesized by the palladium catalyzed Heck coupling reaction. ${ }^{20}$ Compared to other PPV synthetic methods such as Mc Murray condensation, Wittig reaction, and the precursor polymer approach, the mild reaction condition can tolerate a variety of functional groups and hence different types of functionalized PPV can be easily prepared. We have synthesized three types of PPV (polymers A-C) derived from three ruthenium containing monomers (Figures 2-3). These polymers are soluble in common organic solvents and can be processed into optical quality thin film by spin coating or solution casting techniques. For those polymers with lower metal content, they are soluble in non polar solvents such as 1,2-dichloroethane or 1,1,2,2-tertachloroethane. Those polymers with higher ruthenium content are soluble in polar aprotic solvents such as $N, N$-dimethylformamide (DMF) or $N$-methylpyrrolidinone (NMP). 


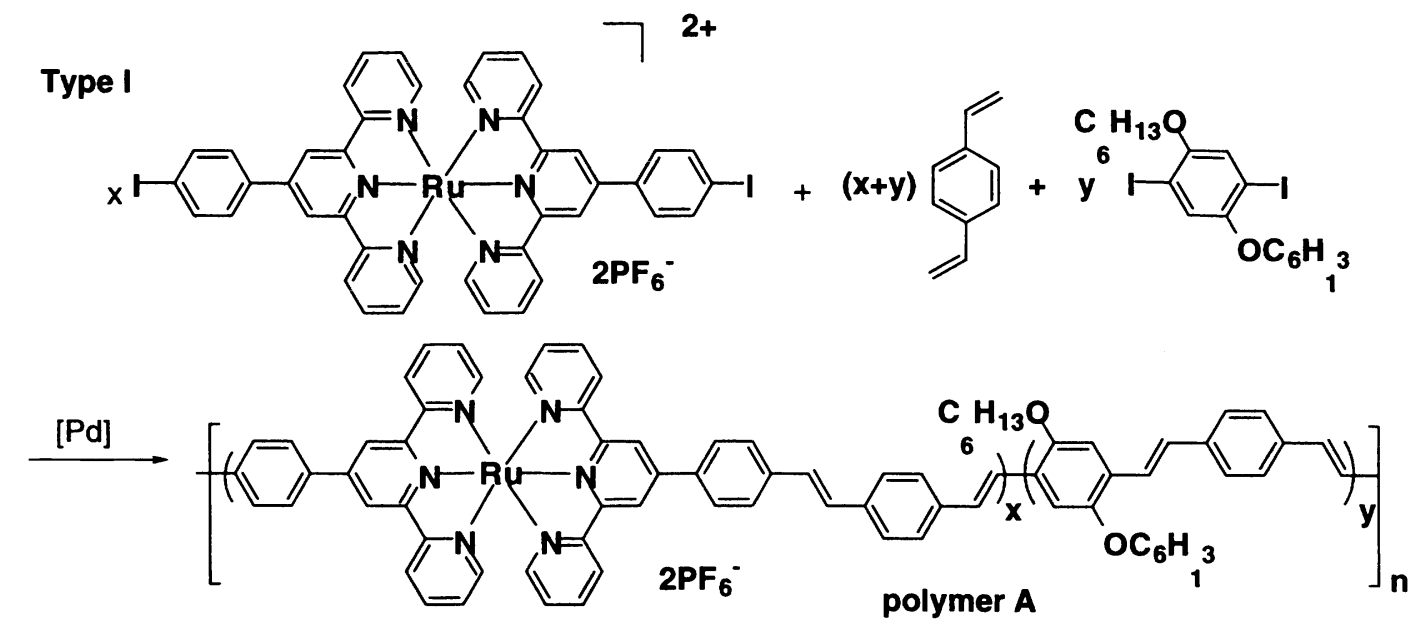

Figure 2. Synthesis of ruthenium functionalized polymer $\mathbf{A}$ with ruthenium center as part of the main chain.

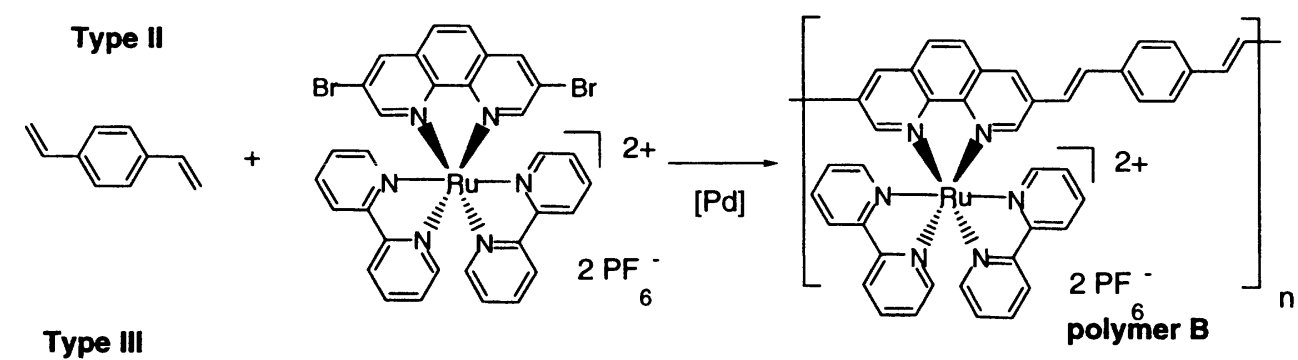

$(x+y)$

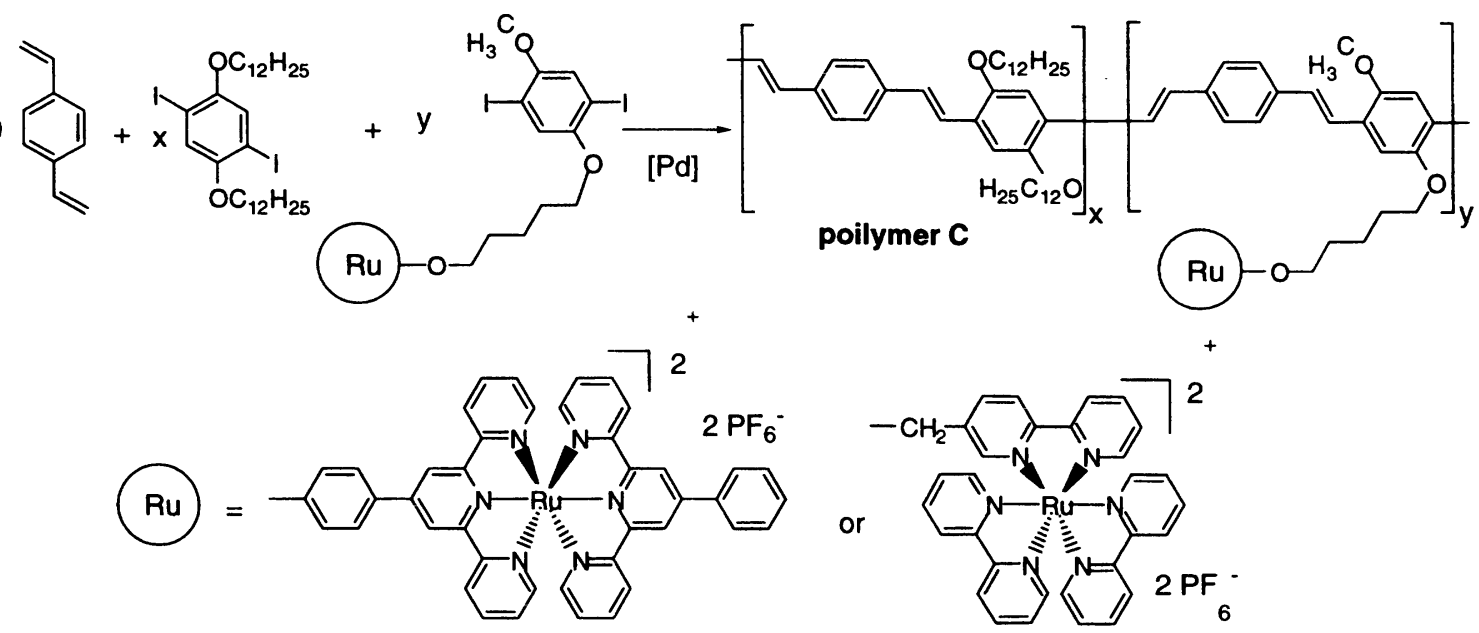

Figure 3. Syntheses of polymers $\mathbf{B}$ and $\mathbf{C}$ with the ruthenium polypyridine complexes attached to the polymer main chain or side chain. 


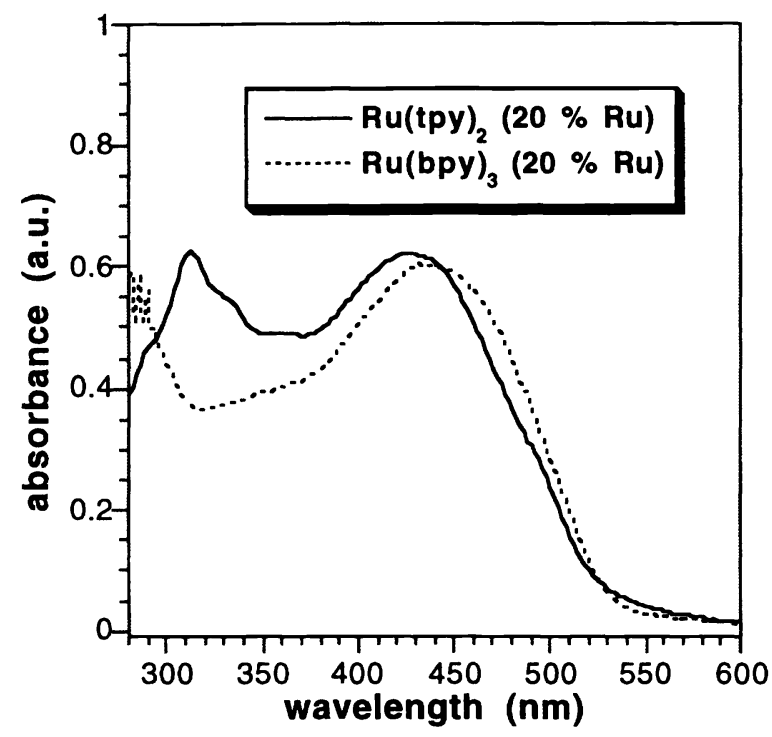

Figure 4. Electronic absorption spectra of polymer $\mathbf{C}$ with pendant ruthenium bipyridine or terpyridine complexes.

The electronic absorption spectra of these polymers mainly consist of two components. Two strong absorption bands are usually observed at ca. 290 and $460 \mathrm{~nm}$ which are due to the $\pi$ - $\pi^{*}$ transition of the polypyridine ligand and the $\pi$-conjugated mainchain, respectively. In addition, there is also another absorption peak centered at the vicinities of 500-550 $\mathrm{nm}$ which is assigned to be the metal-to-ligand charge transfer transition. It is considered to be the promotion of an electron from the $d \pi(\mathrm{Ru})$ to the $\pi^{*}(\mathrm{bpy}$ or tpy) orbitals, and is a characteristic absorption features for ruthenium polypyridine complexes. Figure 4 shows the typical absorption spectra for polymer $\mathrm{C}$ functionalized with pendant ruthenium bipyridine and terpyridine complexes. The spectra are dominated by the absorption due to the conjugated main chain, while the absorption due to the metal complex only appears as a small shoulder. However, in the PL spectra, the intensities of the emissions originated from the MLCT excited states are comparable to those from the main chain.

The charge carrier mobilities of these polymers were studied by the conventional time-of-flight technique. A polymer film with thickness of approximately $1 \mu \mathrm{m}$ was prepared by solution casting on an indium-tin-oxide (ITO) coated glass slide. A layer of semi-transparent gold electrode (thickness $\sim 100 \AA$ ) was coated on the polymer surface by evaporation under high vacuum. A nitrogen laser $(337 \mathrm{~nm}$, pulse width $=5 \mathrm{~ns})$ was used to generate a sheet of charge carriers. Under an externally applied electric field, the movement of charge carriers was monitored with an oscilloscope. The drift mobility $\mu$ was calculated according to the equation $\mu=L / t_{T} E$ where $L$ is the film thickness, $E$ is the applied electric field, and $t_{T}$ is the transit time which corresponds to the time when the leading part of the carrier distribution reaches the opposite electrode. The transient photocurrent profile indicates a dispersive charge transport with non-Guassian carrier distribution. The charge carrier mobilities under different applied field strength and temperature were also studied. An Arrhenius plot of the charge mobility at different temperature indicates a thermally activated charge migration process and the activation energies were estimated to be in the range between 0.1 to $0.2 \mathrm{eV}$. 


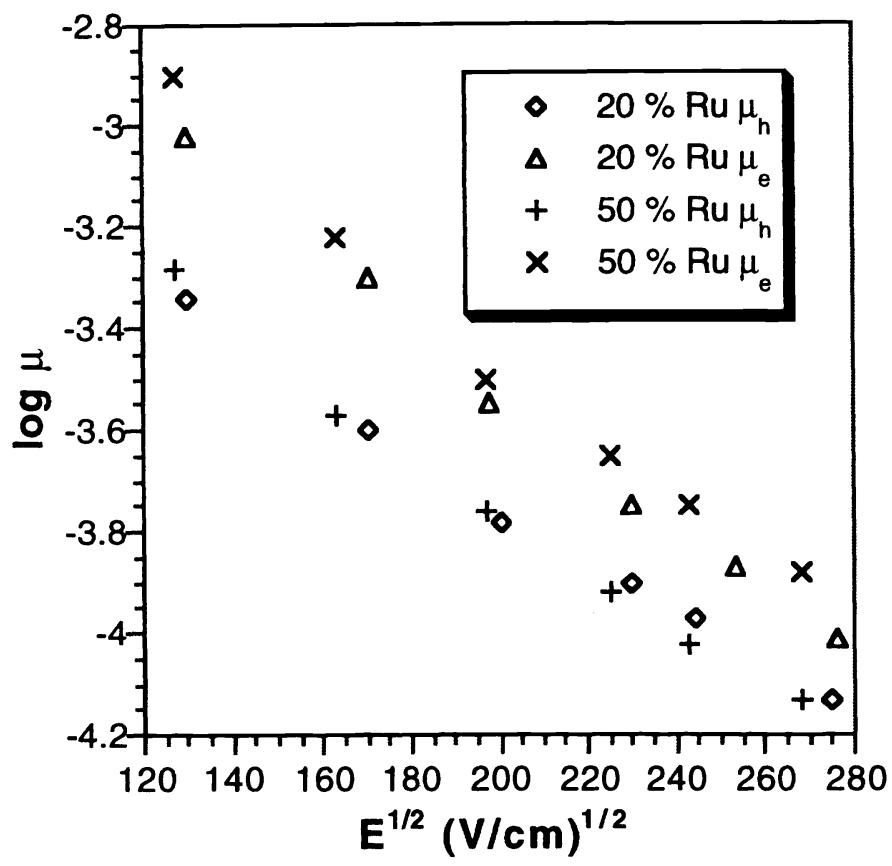

Figure 5. Field dependence hole and electron carrier mobilities for polymer B with different ruthenium content.

The field dependent charge carrier mobilities show a very interesting feature. A plot of hole mobility at different applied electric field strength for polymer B with different ruthenium content is shown in Figure 5 . The electron carrier mobilities $\mu_{e}$ of the polymers are in general higher than the hole carrier mobilities $\mu_{h}$. Those polymers with higher ruthenium content also exhibit higher mobilities. This is probably due to the fact that the ruthenium bipyridine complexes are able to enhance the carrier mobility by providing more charge carrier to the polymers. Since an electric field reduces the barrier for jumps along the field direction, the drift mobility is expected to increase with increasing field strength. However, the $\log \mu$ vs. $E^{1 / 2}$ plot shows a nearly linear relationship with negative slope, indicating that increasing the electric field would decrease the carrier mobility. This interesting phenomenon can be explained by the presence of off-diagonal disorder in the hopping sites. There are enhanced routes for charge transport which involve some short hops not in the direction of the electric field. ${ }^{21}$

The electroluminescence behavior of the polymers were evaluated by preparing a simple single layer light emitting device with the configuration ITO/polymer/Al. Typical polymer film thickness is approximately 50-70 $\mu \mathrm{m}$. The device is subjected to forward bias with the ITO as the anode and aluminium as the cathode. The current voltage characteristics of the polymer $\mathbf{C}$ with pendant ruthenium bipyridine or terpyridine complexes are shown in Figure 6 . Both systems show typical diode character and exhibit turn-on voltage at $5 \mathrm{~V}$ with rectification ratio greater than 1000 at $15 \mathrm{~V}$. The external quantum efficiencies of the devices are estimated to be $0.1 \%$, and the maximum luminance of the device was approximately $360 \mathrm{~cd} / \mathrm{m}^{2}$. 


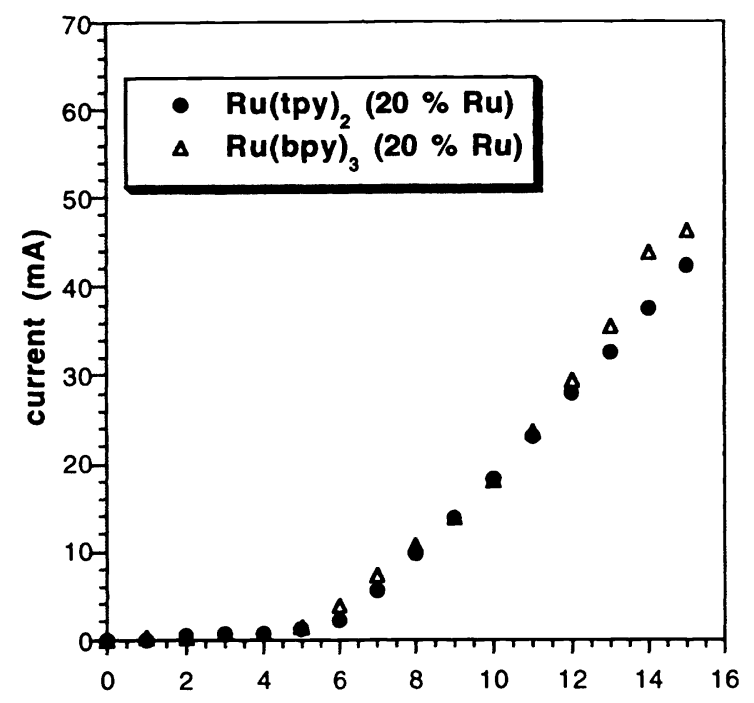

Figure 6. Current-voltage characteristic of the light emitting devices fabricated from polymer $\mathbf{C}$ with pendant ruthenium bipyridine or terpyridine complexes.

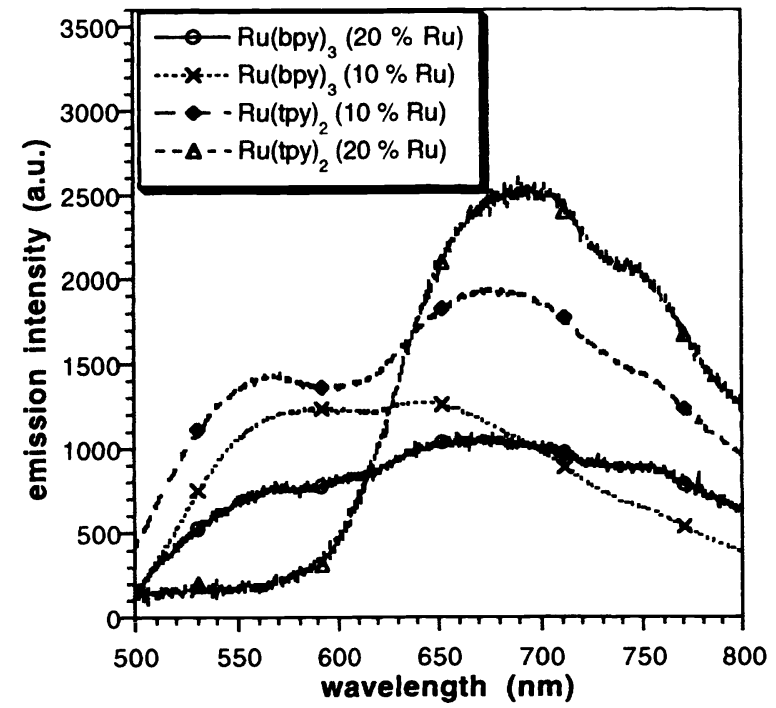

Figure 7. Electroluminescence spectra of the light emitting devices fabricated from polymer $\mathbf{C}$ with pendant ruthenium bipyridine or terpyridine complexes under a forward bias of $20 \mathrm{~V}$. 
Figure 7 shows the EL spectra of polymer $\mathbf{C}$ with pendant ruthenium bipyridine and terpyridine complexes under the forward bias condition. Two emission bands at 560 and $650 \mathrm{~nm}$ are observed in the ruthenium terpyridine containing polymers whose intensity ratio correlates to the $\left[\mathrm{Ru}(\mathrm{tpy})_{2}\right]^{2+}$ content in the polymer. Similar emission properties are also observed in the ruthenium bipyridine containing polymers with $10 \%$ ruthenium except that the peak of the MLCT emission shifts to $680 \mathrm{~nm}$. In these polymers, the resulting yellow light originates from the simultaneous emission from the PPV backbone (green light) and the ruthenium polypyridine complexes (red light). For the polymer which contains $20 \%\left[\mathrm{Ru}(\mathrm{bpy})_{3}\right]^{2+}$ complex, however, only one red emission peak is found at $690 \mathrm{~nm}$. We attribute this interesting phenomenon to the energy transfer between the ruthenium bipyridyl complexes and the polymer mainchain. When the metal complex content is low, quenching of the backbone is not efficient because the backbone and the metal complex moieties are separated by a hexamethylene spacer linkage. When the $\left[\mathrm{Ru}(\mathrm{bpy})_{3}\right]^{2+}$ content is increased to 20 $\%$, the quenching process becomes dominant. It can also be seen that the $\left[\mathrm{Ru}(\mathrm{tpy})_{2}\right]^{2+}$ complex is not an efficient quencher compared to the bipyridyl analog. It is not fully understood why there was no quenching in the PL process. The difference between PL and EL spectra may suggest that these two processes undergo different mechanisms. Nevertheless, these results demonstrate that the emission color of the polymers can be tuned by adjusting the metal complex content or by using different transition metals. In addition, by preparing mix-metal complexes, it is also possible to prepare multicolor display devices.

The schematic diagram for the energy transfer process is shown in Figure 8. Upon excitation, the $\pi$-conjugated backbone can either undergoes light emission $\left(\pi^{*}-\pi\right)$ or an energy transfer process in which its energy is transferred to the metal complexes. The excited state of the metal complex can subsequently undergoes light emission from the MLCT excited states. As a result, the emission from the conjugated mainchain is apparently quenched by the metal complexes, and the resulting color of light emission is dependant on several factors such as the distance between the metal and main chain, nature of the spacer group, and overlap of the absorption and emission spectra etc. It was found that in the derivatives of polymer $\mathbf{B}$, this quenching process is more efficient. Only red light emission was observed when the ruthenium content was $20 \%$. By understanding the detail of this energy transfer process, it is possible to tune the emission color by simply varying the metal content without modifying the structure of the polymers.

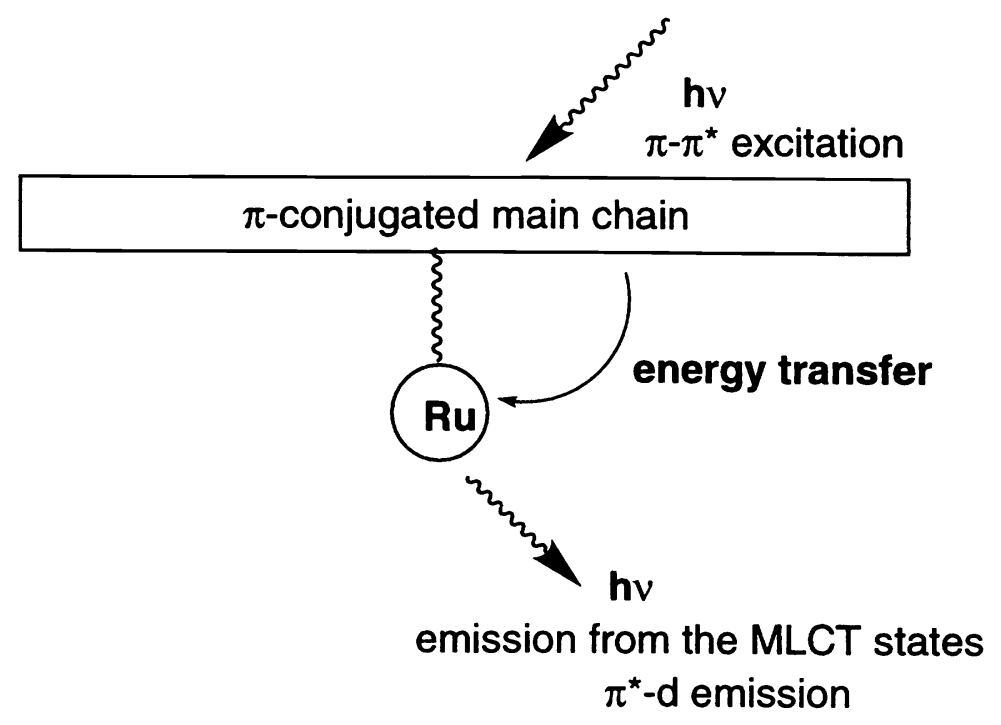

Figure 8. Schematic diagram representing the energy transfer process in the ruthenium containing polymers. 


\section{CONCLUSIONS}

We have developed different series of conjugated polymers functionalized with ruthenium bipyridine or terpyridine complexes on the polymer main chain or side chain. It was found that the content and the nature of the metal complexes might affect the physical properties of the polymers such as photosensitivity, charge carrier mobilities, and luminescence properties. Moreover, the metal complexes are able to quench the emission from the $\pi$-conjugated main chain and it is possible to tune the emission color by varying the structure or metal content of the polymers.

\section{ACKNOWLEDGMENTS}

This work was supported by the Research Grants Council of The Hong Kong Special Administrative Region, China (Project No. HKU 7093/97P and HKU 7090/98P) and The University Research Committee, University of Hong

Kong. Partially financial support from the Hung Hing Ying Physical Science Research Fund and the Run Run Shaw/Leung Kau Kui Research and Teaching Endowment Fund is also acknowledged.

\section{REFERENCES}

1. J. H. Burroughes, D. D. C. Bradley, A. R. Brown, R. N. Marks, K. Mackay, R. H. Friend, P. L. Burns, and A. B. Holmes, Nature 347, pp. 539, 1990.

2. G. Gustafsson, Y. Cao, G. M. Treacy, F. Klavetter, N. Colaneri, and A. J. Heeger, Nature 357, pp. 477, 1992.

3. N. C. Greenham, S. C. Moratti, D. D. C. Bradley, R. H. Friend, and A. B. Holmes, Nature 365, pp.628, 1995.

4. J. Kido, K. Hongawa, K. Okuyama, and K. Nagai, Appl. Phys. Lett. 64, pp. 815, 1994.

5. T. A. Hopkins, K. Meerholz, S. Shaheen, M. L. Anderson, A. Schmidt, B. Kippelen, A. B. Padias, H. K. Hall, N. Peyghambarian, and N. R. Armstrong, Chem. Mater. 8, pp. 344, 1996.

6. J. Kido, H. Hayase, K. Honogawa, K. Nagai, and K. Okuyama, Appl. Phys. Lett. 65, pp. 2124, 1994.

7. D. L. Thomsen III and F. Papadimitrakopoulos, Polymer Preprint 38(1), pp. 398, 1997.

8. J.-K. Lee, D. Yoo, and M. F. Rubner, Chem. Mater. 9, pp. 1710, 1997.

9. W. Y. Ng and W. K. Chan, Adv. Mater. 9, pp. 716, 1997.

10. W. K. Chan, X. Gong and W. Y. Ng, Appl. Phys. Lett. 71, pp. 2919, 1997.

11. S. C. Yu, X. Gong and W. K. Chan, Macromolecules 31, pp. 5639, 1998.

12. X. Gong, P. K. Ng, W. K. Chan, Adv. Mater. 10, pp. 1337, 1998.

13. C. T. Wong, W. K. Chan, Adv. Mater. 11, pp. 455, 1999.

14. K. Kalyanasundaram, Coord. Chem. Rev. 46, pp. 159, 1982.

15. A. Juris, V. Balzani, F. Barigelletti, S. Campagna, P. Belser, A. Von Zelewsky, Coord. Chem. Rev. 84, pp. 85, 1988.

16. E. C. Constable, Adv. Inorg. Chem. Radiochem. 30, pp. 69, 1986.

17. E. C. Constable, J. Lewis, M. C. Liptrot, P. R. Raithby, Inorg. Chim. Acta. 178, pp. 47, 1990.

18. J.-P. Sauvage, J.-P. Collin, J.-C. Chambron, S. Guillerez, C. Coudret, V. Balzani, F. Barigelletti, L. De Cola, L. Flamigni, Chem. Rev. 94, pp. 993, 1994.

19. W. Helfrich, W. G. Schneider, J. Chem. Phys. 44, pp. 2902, 1966.

20. R. F. Heck, Organic Reactions 27, pp. 345, 1982.

21. M. Van der Auweraer, F. C. De Schryver, P. M. Borsenberger, H. Bässler, Adv. Mater. 6, pp. $199,1994$. 